\title{
Space Network Modeling and Simulating in Space-Ground Integrated Networks
}

\author{
Qi Guo a, Ruxin Zhi ${ }^{\text {b }}$, Beishan Wang and Bo Sun \\ School of Information and Electrics, Beijing Institute of Technology, Beijing, 100081, China \\ aguoqi_bit@163.com, bzhiruxin@163.com
}

Keywords: integrated network, architecture research, space network.

\begin{abstract}
Space-Ground Integrated Networks (SGIN) are systems that extend from terrestrial network to space network, to build a network covering ground and space. This paper firstly introduces the development of SGIN, and analyzes three architectures of SGIN based on existing systems. Afterwards, we focus on space networking and simulate two networks on OMNeT++, whose space network architectures differ in networking among satellites. We examine the system characteristics of different structures by analyzing packet delay and arrival rate, showing that different ways of networking among satellites differ in capacity and application scenes. Finally, the impact of our work on practical construction and future challenges for SGIN are given.
\end{abstract}

\section{Introduction}

With an increasing demand for comprehensive data service all around world and space, space communication has become a considerable option for future networks, and Space-Ground Integrated Network (SGIN) has received marvelous attention recent years. SGIN can achieve a global coverage, making it possible to provide different users with reliable and real-time service at anytime anywhere.

Owing to the advantage in coverage, mobile access and complementary relationship with terrestrial networks, space networks trend to be integrated with terrestrial network to provide users with global Internet access services. According to actual situation and future development trends, SGIN is summarized in [3] as followed: based on terrestrial networks and stretched to space networks, SGIN can achieve coverage all over ground, ocean, air, and space, providing all types of users' activities with communication infrastructure in various areas.

As investigated in $[4,5]$, structures of SGIN are developed in 3 phases: space-satellite-terrestrial network, space-based network, and space-terrestrial network [3]. Space-satellite-terrestrial network is now in widely used (Inmarsat, Globalstar, etc. [6]), who realizes globalization by ground stations distributed all around world while satellites merely play roles as transparent forwarding channel. Space-based network (Iridium, etc.) adopts networking among satellites [7], which makes the space network can operate independently off the ground network. This architecture requires for a complex space network, as functions like handling, controlling, and exchanging information are all accomplished on satellites. Space-terrestrial network (TAST) is a combination of space-based network and terrestrial network. Since space-based network can get a global coverage, there is no need to deploy ground stations all around the world [8] in this structure. Besides, network management and control could be done on the ground.

In this section, we primarily analyze the status quo of SGIN. The rest of the paper is organized as follows. Section 2 introduces the modeling of the two SGIN scenarios, network topology and routing protocols are given. Based on the simulation on OMNet++, section 3 analyzes the simulation results. Section 4 gives the conclusion and future works.

\section{Network Modeling}

We propose 2 SGIN structures based on Beidou Navigation Satellite System from [9], for the system is capable of providing worldwide location information and sending short messages between 
navigation terminals. Via modeling of network topology, protocol flow, node structure and message, simulation of data service access capability in space network can be achieved.

\subsection{Network Architecture.}

Space-based backbone network

Scenario 1 simulates the scene in ground station receiving remote sensing satellite data [10]. This system is composed of three 3 types of nodes: 3 GEO satellites (at the height of $35786 \mathrm{~km}$ ) with uniform distribution to achieve global coverage, a LEO satellite with an orbital inclination of $86.4^{\circ}$ at the height of $780 \mathrm{~km}$ and a ground station (GS) located in Kashi. LEO satellite simulates low-orbit remote sensing satellite, data captured from which need to be transmitted to GS via GEO network.

Space-terrestrial network

Scenario 2 simulates the scene in which users communicate with each other via space network [11]. In this scene, 2 terminals on earth send data packets to each other via space network made up of $24 \mathrm{MEO}$ satellites evenly distributed in 3 orbital planes with different orbit inclination in the height of $27906 \mathrm{~km}$, whose inter-satellite routing is established via an improved OSPF protocol.

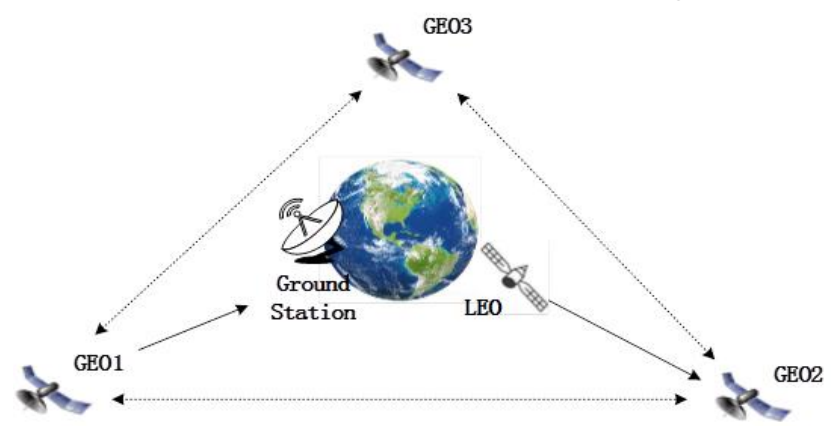

Fig.1 Space-based backbone network

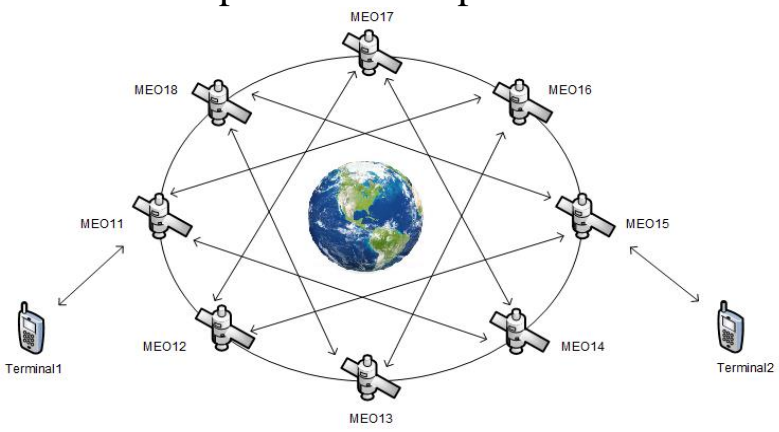

Fig. 2 Space-terrestrial network

Since communication satellites, relay satellites and navigation satellites currently are still in the dedicated network phase [8], the systems cannot achieve an effective sharing of internetworking. Therefore, we simulate and analyze the above two systems separately.

\subsection{Network Protocol Design.}

Our work concentrates on the architectures, thus the routing are based on the existing protocols. According to the TCP/IP hierarchical architecture, the functions of GEO and MEO satellites are mainly data relay, so the modeling of these nodes includes physical layer, link layer, network layer and some function modules, and the upper layer protocols are weakened. For LEO remote sensing satellite, it involves the generation of data and sending, thus the modeling includes all the five layers.

OSPF (Open Shortest Path First) is an interior gateway protocol used for deciding routing in a single autonomous system. Here we use an improved OSPF protocol to establish inter-satellite routing among 24 MEO satellites, for that using OSPF in satellites requires an increase in the update cycle. Due to the restriction of satellite antenna angle, each satellite has three point-to-point inter-satellite links, of which two are the same orbit satellite link (shown in Fig.2), one for other orbit satellite link.

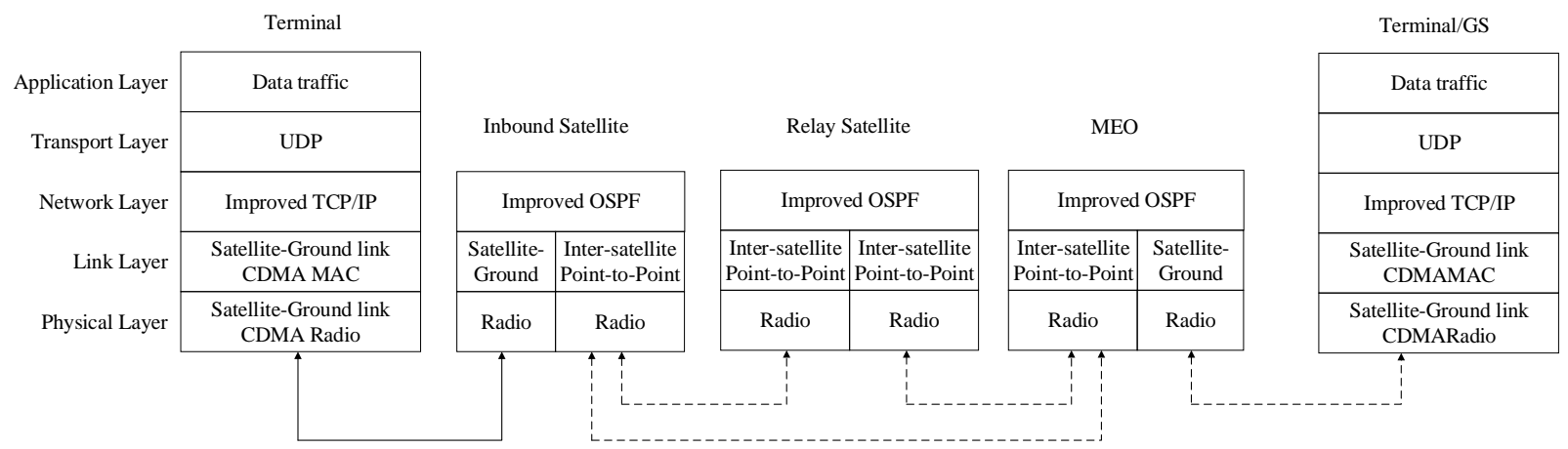

Fig.3 Protocol stack design of each simulation nodes 


\subsection{Simulation Description.}

Simulations of abovementioned networks are based on discrete event simulator OMNeT++, which is friendly-using and powerful to model wireless communication networks. To evaluate the systems, we investigate packet delay (end-to-end delay, which sums transmission delay, propagation delay and processing delay) and arrival rate (delivery ratio) [10] to demonstrate the transmission characters.

In scenario 1, GEO satellites are relatively stationary to the ground, GS is only in the visible range of GEO1, thus LEO would transmit the data via LEO-GEO1-GS, or LEO-GEO2/GEO3-GEO1-GS. We simulate this scenario in duration of 100,000 s. LEO generates a packet on average per second, with a length varying from $100 \mathrm{~B}$ to $10 \mathrm{MB}$. The inter-satellite link frequency is set at $30 \mathrm{GHz}$ and the bandwidth is set to $1.2 \mathrm{GHz}$, and LEO, GEO, GS access rates are: $622 \mathrm{Mbps}, 1.2 \mathrm{Gbps}, 155 \mathrm{Mbps}$

In scenario 2, channel matching should be done before issuing the data service. When application layer service occurs from the terminal, data is cached at MAC layer as soon as the packet arrives. Both sending and receiving terminal broadcast the channel assignment request to all visible MEO satellites, afterwards, each MEO received the request sends back the terminal a reply frame. Once the reply is received, terminal sends a confirmation frame, immediately the terminal and a certain MEO satellite establish a connection through the allocated codeword. Meanwhile, satellite-ground link is added to the terminal and MEO routing table. We simulate this scenario in duration of 5,000s. terminals send packet per second to each other, with a length varying from 100B to 100MB. Other parameters are set similar to scenario 1.

\section{Analysis of the Results}

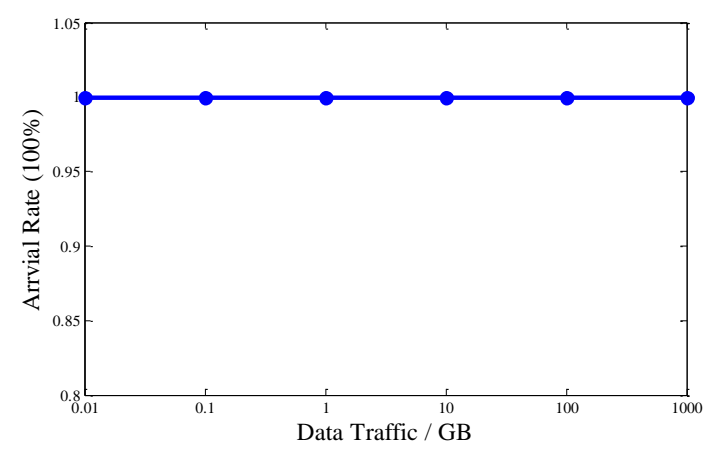

Fig.4 Arrival Rate

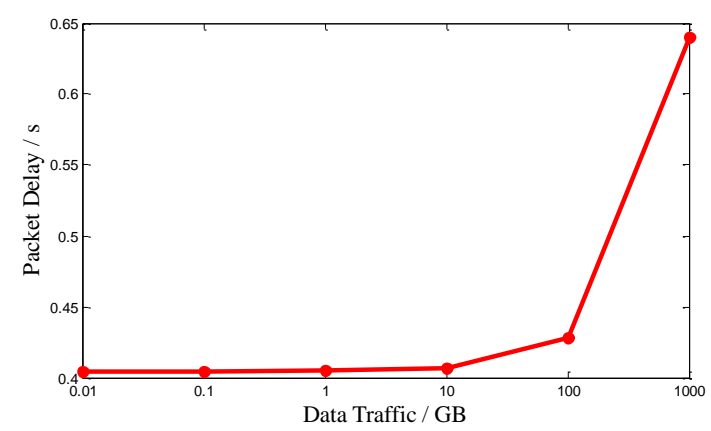

Fig.5 Average Packet Delay

Figures 4 and 5 show the results of GEO-SGIN. With communication among the GEO satellites, LEO is visible to space network all the time, data transmission can be properly conducted periodically, thus arrival rate is always close to 1 (0.9995). When total data traffic increases, transmission still functions correctly as shown in Fig.4, while the average transmission delay rises, resulting in the endto-end delay increasing in Fig.5.

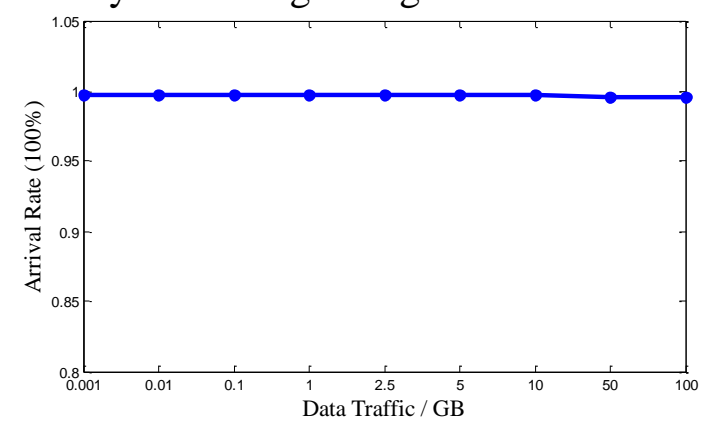

Fig.6 Arrival Rate

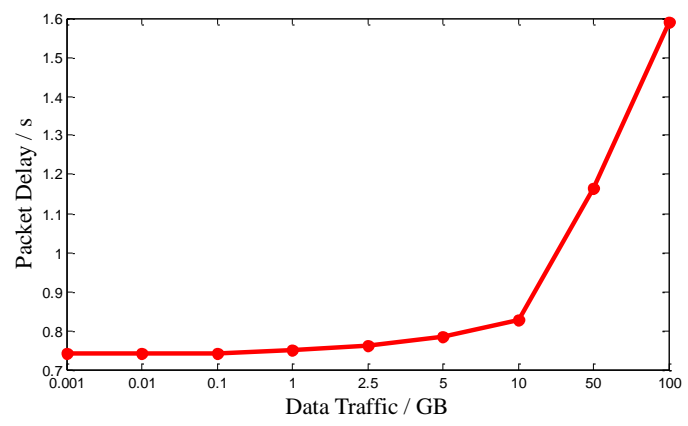

Fig.7 Average Packet Delay

Figures 6 and 7 show the results in simulating scenario 2, and the overall trend is similar to scenario 1. To establish the link among satellites and terminals, the very first packet of each link is lost, so the average delivery rate is a little lower (0.997). As for the time delay, MEO network is less in satelliteground link, however, scenario 2 hops more in satellite links, reaching an average at 0.76 s making 
the total delay longer than scenario 1. With data traffic increasing, time delay increases greatly, indicating a relatively worse data access capability.

Considering the practical application, GEO satellites in a certain system can hardly achieve a worldwide distribution, which keeps the data cached during the time LEO is invisible to GEO, causing a longer time delay. Besides, if GEO space-based network is used in ground terminal communication, it can no longer achieve a real sense of global coverage. Apart from this, since the orbit height is much higher, handheld terminals would require for a higher antenna power to send data. Hence, MEO space-terrestrial network works better in global terrestrial communication. However, GEO satellites are usually bigger than MEO satellites, which indicates a relatively better data processing capability and system capacity.

\section{Conclusion}

In this paper, we simulated 2 Space-Ground Integrated Networks. In general, GEO-SGIN network is functionally simple, easy in infrastructure construction, but limited in application range. MEO based network has a stable routing, and works effectively in global communication. However, it calls for more on connections within satellites, causing system maintenance difficulties to a certain extent.

With the high pace of Space-Ground Integrated Network, single-layered space network as described in this paper would no longer meet the communication needs in the future, therefore, space network in different height should be combined together to achieve a stable real-time communication.

\section{Acknowledgements}

This project is supported by 863 project, project number 2015 AA015701.

\section{References}

[1]. Harvey Newman, M. Spiropulu, J. Balcas, et al. Next-Generation Exascale Network Integrated Architecture for Global Science [Invited]. IEEE/OSA Journal of Optical Communications \& Networking, Vol.9(2017) N0.2, p.A162-A169.

[2]. Hao Wu, Jian Li, Hancheng Lu, Peilin Hong. A Two-Layer Caching Model for Content Delivery Services in Satellite-Terrestrial Networks. 2016 IEEE Global Communications Conference (GLOBECOM), Washington DC, United Srates, December 4-8, 2016, pp. 1-6.

[3]. Hewu $\mathrm{Li}$, Qian $\mathrm{Wu}, \mathrm{Ke} \mathrm{Xu}$, et al.Progress and tendency of space and earth integrated network. Science \& Technology Review. Vol.34(2016).No.14,p. 95-106

[4]. Manqing $\mathrm{Wu}$, Wei $\mathrm{Wu}$, Bin Zhou, et al. Tentative Plan for Overall Structure of Integrated Information Network. Satellite and Network. Vol.3(2016).p.30-36.

[5]. Huang. G: Research on hierarchical routing for satellite/terrestrial integrated networks.( MS, University of Science and Technology of China, China, 2015).p.3.

[6]. Ya Wang, Lidong Zhu. Development and Application of Space Internet. Space International. Vol.3(2016), p. 43-49

[7]. Xiangge Liu, Lei Shi, Wenxi Wu. Based on space-ground integrated information network of data assimilation analysis. International Symposium on Communications and Information Technologies, Qingdao, China, November 21,2016, p.487-493.

[8]. Mingchuan Yang, Xinye Shao, Zhongzhao Zhang, et al. Research on Architecture and Key Issue of Integrated/Hybrid Satellite-Terrestrial Systems. Academicals Annual Conference on Satellite Communications, Beijing, China, 2016, p.46-55

[9]. Phil Liebrecht, James Schier, Kul B. Bhasin, et al. NASA's integrated space communications architecture. SpaceOps 2010 Conference Delivering on the Dream Hosted by NASA Marshall Space Flight Center and Organized by AIAA, Huntsville, AL, United States, April 25-30, 2010, p.1-12. 
[10]. Junlin Wang, Chunsheng Liu. Development and Application of INMARSAT Satellite Communication System. First International Conference on Instrumentation, Measurement, Computer, Communication and Control, Beijing, China, October, 21-23, 2012, pp.619-621.

[11]. Ge Xu, Min Sheng, Chengke Wu. Advanced Routing In Interplanetary Backbone Network. Second International Conference on Spatial Information Technology, Wuhan, China, November 10-11,2007, p.679513-1-6. 